\title{
Response to the Letter to the Editor by Rob Armstrong
}

\author{
Robert H. Six ${ }^{1 *}$, David R. Young ${ }^{2}$, Melanie R. Myers ${ }^{1}$ and Sean P. Mahabir ${ }^{1}$
}

\begin{abstract}
In a recent Letter to the Editor, Armstrong raises concern that the design of the study reported by Six et al. was not consistent with the product label for treatment of Amblyomma americanum, since fluralaner was not re-administered 56 days after the initial treatment. The Authors disagree with this assessment and confirm that the design was appropriate, and therefore the results and conclusions for the entire study period are valid.
\end{abstract}

\section{Letter to the Editor}

Six et al. reported the results of a study demonstrating that monthly administration of sarolaner consistently killed significantly more $A$. americanum ticks within $24 \mathrm{~h}$ than a single dose of fluralaner from 6 weeks after initial treatment [1]. In his Letter to the Editor, Armstrong raised concern that the efficacy of fluralaner was evaluated at times outside the label administration recommendations [2].

We appreciate the author's review and comments on the manuscript; however, there are several points that need to be addressed in order to appropriately evaluate the conclusions they have drawn:

(i.) The author states that the approved Bravecto $0^{\circ}$ label requires that fluralaner be re-administered at 56 days for the treatment $A$. americanum when in fact the label $[3,4]$ states that fluralaner may be re-administered every 8 weeks in case of potential exposure to $A$. americanum (although the general dosing directions state fluralaner should be administered every 12 weeks). This language is very confusing and may be interpreted by a pet owner or veterinarian to mean that fluralaner may be effective against $A$. americanum for up to 12 weeks. This supports the study design which evaluated efficacy for 12 weeks and therefore we believe the results and conclusions for the entire study period are valid.

\footnotetext{
* Correspondence: robertsixwork@comcast.net

${ }^{1}$ Zoetis, Veterinary Medicine Research and Development, 333 Portage St,

Kalamazoo, MI 49007, USA

Full list of author information is available at the end of the article
}

(ii.) The author states that the manuscript reports in the Discussion section that the Bravecto ${ }^{\circ}$ label directions were not followed when in fact the Discussion section simply references the efficacy of fluralaner against $A$. americanum as reported in the Bravecto ${ }^{\circ}$ Freedom of Information Summary [3]; the design of the study (a single dose of fluralaner compared to 3 monthly doses of Simparica ${ }^{\circ}$ over a 90 day period) is clearly stated in the Methods section of the Abstract.

(iii.) The author accurately summarizes the manuscript which reported that at the 8 and $12 \mathrm{~h}$ counts on Day 42 the efficacy of both products was lower than expected for unknown reason; however, at the $24 \mathrm{~h}$ count on Day 42 and for the duration of the study through Day 90, sarolaner provided significantly lower live tick counts compared to fluralaner $(P \leq 0.0006)[1]$.

The design, conduct and reporting of this study are therefore accurate and appropriate. Under the conditions of this study, monthly administration of Simparica provided consistently high (>70\%) efficacy against $A$. americanum within $24 \mathrm{~h}$ of re-infestations over the 3month ( 3 dose) period and from Day 42 onwards this efficacy was significantly better than that provided by fluralaner.

Veterinarians and pet owners cannot predict at the time of treatment the genera and species of tick to which a dog will potentially be exposed. This consideration is of even greater importance when a long-acting product (where the tick genera and species must be predicted months in advance) is used. With Bravecto ${ }^{\circ}$ 
this is further complicated by the fact that the duration of efficacy and therefore the ideal treatment interval varies depending upon the tick species. The design of the study is consistent with the fluralaner re-treatment interval recommended on the product label (anywhere between 8 and 12 weeks), and demonstrates the efficacy observed under controlled conditions from a re-treatment interval that will possibly be used on dogs exposed to A. americanum.

This study therefore clearly demonstrates that the rapid and consistent kill of A. americanum provided by monthly dosing with Simparica ${ }^{\circ}$ will reduce the direct deleterious effects of tick feeding and should reduce the risk of tickborne disease transmission better, and more reliably than a single dose of fluralaner over a 90-day period.

\section{Acknowledgements}

The authors would like to thank Steven Maeder for his assistance in preparing this Letter.

\section{Funding}

This study was funded by Zoetis, Parsippany, NJ.

\section{Availability of data and materials}

Data upon which conclusions are based were presented in the paper.

\section{Authors' contributions}

All authors assisted with the design and conduct of the study, interpretation of the data, and manuscript review. DRY conducted the study, which was monitored by MRM. SPM conducted the statistical analyses. All authors read and approved the final version of the manuscript and this letter.

\section{Competing interests}

This study was funded by Zoetis, Florham Park, NJ. RHS, MRM and SPM are current employees of Zoetis. DRY was an investigator contracted for this study.

\section{Consent for publication}

Not applicable.

\section{Ethics approval}

The protocol was reviewed and approved by the YVRS Institutional Animal Care and Use Committee prior to implementation.

\section{Publisher's Note}

Springer Nature remains neutral with regard to jurisdictional claims in published maps and institutional affiliations.

\section{Author details}

'Zoetis, Veterinary Medicine Research and Development, 333 Portage St, Kalamazoo, Ml 49007, USA. ${ }^{2}$ YVRS, 7243 East Ave, Turlock, CA 95380, USA.

Received: 5 April 2017 Accepted: 18 April 2017

Published online: 26 April 2017

\section{References}

1. Six RH, Young DR, Myers MR, Mahabir SP. Comparative speed of kill of sarolaner (Simparica ${ }^{\mathrm{TM}}$ Chewables) and fluralaner (Bravecto ${ }^{\circ}$ ) against induced infestations of Amblyomma americanum on dogs. Parasit Vectors. 2016;9:399.

2. Armstrong R. The conclusion of a comparative efficacy study of fluralaner and sarolaner against the tick Amblyomma americanum on dogs is based on results obtained at study times that are outside the fluralaner label recommendations. Parasit Vectors. 2017;10:159.

3. FDA, Package Insert. BRAVECTO. http://us.bravecto.com/pdfs/bravecto_pi_ mah.pdf. Accessed 29 Sept 2016.

4. FDA, FOI. NADA 141-426. BRAVECTO. http://www.fda.gov/downloads/ animalveterinary/products/approvedanimaldrugproducts/ foiadrugsummaries/ucm399075.pdf. Accessed 20 Aug 2016.

Submit your next manuscript to BioMed Central and we will help you at every step:

- We accept pre-submission inquiries

- Our selector tool helps you to find the most relevant journal

- We provide round the clock customer support

- Convenient online submission

- Thorough peer review

- Inclusion in PubMed and all major indexing services

- Maximum visibility for your research

Submit your manuscript at www.biomedcentral.com/submit 